\title{
Identification and management of problem eaters
}

\author{
David Skuse
}

Investigations into the prevalence of feeding problems among preschool children have found a remarkably high proportion are affected. Minde and Minde, reviewing the available epidemiological data, discovered that estimates of between $12-34 \%$ were usual. ${ }^{1}$ The sorts of difficulties that present to paediatricians tend to fall into two broad categories: refusal to eat (often found in association with impaired growth) and excessive faddiness. However, some feeding difficulties that have developmental consequences during infancy are more subtle than this.

Many toddlers who decline to eat a normal diet, in terms of quality and quantity, have been difficult feeders from an early age. Often the history one obtains is of a longstanding fussiness, a resistance to try other foods, bouts of colic, and sometimes self induced vomiting in association with mealtimes. The management of specific conditions such as infantile colic, gastro-oesophageal reflux, rumination, feeding difficulties associated with structural defects of the oropharynx, and gastric motility problems are topics outside the scope of this review. Instead, the emphasis will be upon the identification and management of feeding problems of infants and preschool children that are relatively common, but are either missed by the families' medical attendants or are mishandled.

\section{Sensitive periods: taste}

Recent work has suggested that there could be a sensitive period, between about 4-6 months of age, during which infants will usually accept any new taste. If they have a number of exposures to that taste a preference may be induced that persists into later childhood. ${ }^{23}$ This is an intriguing hypothesis, for normally solid food is liable to be introduced, in most Western cultures, at about four months postterm from nutritional necessity. ${ }^{4}$ So there may be a window of opportunity, between about four and six months, when infants will accept just about any taste that is presented to them and tolerance of these tastes will persist into later childhood.

If food preferences really are conditioned by exposure in the early months, and we must not forget that a range of strong tasting foods in a mother's diet (for example, curries) will influence the taste of breast milk, then to delay introduction of a wide range of tastes until after six months or so is likely to meet with food refusal, especially by individuals who are temperamentally difficult. There is at least the possibility that culturally determined practices, such as the late weaning found in the Bangladeshi community, ${ }^{5}$ will by their very nature provoke feeding difficulties. When family foods are introduced, perhaps in the latter half of the second postnatal year, anecdotal evidence suggests they tend to be resisted, possibly as a consequence of this developmental process.

\section{Sensitive periods: texture}

For many years it has been acknowledged clinically that there may be a 'sensitive period' for the introduction of solid foods. If children are not exposed to solids that require chewing by about 6-7 months of age they tend to be resistant to accepting such textures in later childhood. ${ }^{6}$ Feeding problems can then result, with refusal to accept lumpy foods, spitting out, and occasionally vomiting. The lack of experience of chewing firm solids may be associated, by a year or so of age, with immature and restricted tongue movements. Consequently firm solids will not be masticated adequately for ease of swallowing. Attempts to swallow will then lead to gagging, which is for the infant a powerful aversive stimulus, after which the child may refuse to ingest anything but liquids and puréed material. Early exposure to a variety of textures seems to be of crucial importance for the development of normal oral motor skills. Yet in hospital practice a significant proportion of very young children are fed for prolonged periods by nasogastric tube. Infants fed by this means are not usually exposed to concurrent oral stimulation and so there is a dramatic reduction in the amount of sensory input and perception in the mouth. The normal infant receives a continuous bombardment of varied oral stimulation through food, sucking fingers, pacifiers, and other objects. When this is reduced because of nasogastric tube feeds the oral cavity can become hypersensitive and hyperirritable. ${ }^{7}$ There may be an associated loss of sucking and swallowing skills, depending on the timing and duration of the artificially delivered feeds. Furthermore, the presence of a tube in the nasopharynx can induce a local low level inflammatory response with excessive secretions, so that many children fed in this way appear to have a persistent mild upper respiratory tract infection. 
One consequence of such nasal congestion is to reduce the sense of taste (smell) to the extent that, even if oral feeds are introduced alongside the nasogastric feeds, there is no gustatory reinforcement for what is otherwise a largely unpleasurable experience. Food refusal is a frequent outcome; the child is likely strongly to resist oral feeds. Body weight may then drop and it is not unusual to find that the nasogastric tube is eventually replaced by a medical team anxious their patient does not succumb to inanition. The appropriate way of handling this situation has been described in detail by Morris ${ }^{7}$; a crucial component of the management of children being fed in this way is to ensure that appropriate oral stimulation is given throughout the period; so-called 'sham feeding'. A comprehensive multidisciplinary approach is required. By failing to address the special needs of non-orally fed infants and young children in our paediatric wards we are doing them and their parents a grave disservice.

\section{Faddiness}

Other things being equal, young children usually show a preference for familiar over novel foodstuffs. Their 'fear' of new tastes and textures has been termed 'neophobia'. There is very little information on the emergence and developmental course of neophobic responses but anecdotal evidence suggests that they become predominant only after the first postnatal year. ${ }^{8}$ Most parents handle the resistance to try new foods appropriately, with firmness and encouragement, and they gradually become an accepted part of the child's diet. Perhaps it is not sufficiently well appreciated that neophobia is a perfectly normal part of any child's developing relationship to the environment, and is in many ways adaptive; from a teleological point of view the phenomenon enhances the chance of the child's survival. Rejection of new foods is usually a transitory phenomenon, which can be reduced by means of repeated exposure. Small quantities should be given regularly without undue coercion, followed by plenty of praise if the child ingests some. It is a mistake for parents to interpret the initial rejection as reflecting the child's immutable dislike of the new food, and consequently to remove it altogether from the child's diet.

As I have already discussed, extreme faddiness in preschoolers may be more frequently encountered among those whose early feeding experiences were of a very bland and limited diet up until at least 6 months of age. This evidence, together with that of neophobia, suggests very young children do not necessarily consume a healthy range of foodstuffs, left to their own devices. In the 1920s and 1930s Clara Davis conducted several pioneering studies on the spontaneous selection of food by infants and young children. ${ }^{910}$ The results of her work have been widely interpreted to mean that, given an appropriate set of choices, infants will instinctively select and consume a balanced diet. Such a broad conclusion was not drawn by Davis, nor should it be inferred from her research - or from that of any other investigator. ${ }^{11}$ She did not, possibly because of the economic recession at the time, give the infants the option of trying desserts, ice cream, pastries, or sweets! There is no doubt that infants really do have a distinct preference for sweeter tastes, ${ }^{12} 13$ although this preference may be modified by subsequent dietary experience. ${ }^{14}$

Parents often use palatable, preferred foods, as rewards for children's performance of desired behaviours. In common parlance that simply means saying 'You can have a sweet if you're good'. A series of experiments was designed to investigate the effects of such practices on the acquisition of food preferences. ${ }^{13}$ The results are clear: repeatedly associating a food with a distinctive social context systematically changes children's preferences, in either a positive or negative direction, depending on the social context employed. By social context I mean, approximately, the implicit value placed upon that food. In order to encourage a preschool child to eat a foodstuff about which they have no strong feelings one way or the other, we might promise the child a non-food reward. Perhaps we offer to read a favourite story to the good girl who eats up all her carrots. Research has shown that this results in a significant negative shift in the child's attitude to that food. ${ }^{15} 16$ The reason for this finding is not entirely clear - but we might suppose the child's reasoning goes 'If mummy has to bribe me to eat this then it can't be very nice' - or, in more technical phraseology the coercive nature of the feeding strategy generates a negative emotion which becomes associated with the food in question. This has relevance for medical practice insofar as we often try to encourage children to take medicines by offering them a treat afterwards; the research suggests by so doing we are virtually guaranteeing the child will be less likely to accept the medicine without a fuss next time.

A corollary of the above finding may be found in a fascinating study that was designed to investigate the effects of the foods themselves as the reward component of a similar contingency. In this instance the desired behaviour was nothing to do with eating, and the reward for that behaviour was a food about which the child had a neutral attitude. Perhaps in order to reward a preschool child for playing quietly for half an hour, so as not to interrupt a busy mother, she offers her daughter a banana at the end of that period. Research has shown that, when repeatedly presented with neutral foods as rewards for a desired (non-eating) behaviour the erstwhile neutral foodstuffs become highly valued by children. ${ }^{17}$ Again, the social context suggests the food must be valuable or it wouldn't be worth giving as a reward.

\section{Force feeding}

I believe it can be stated categorically that no child has been induced to increase his or her oral intake of food by force feeding. Yet this 
tactic is often resorted to by mothers and, if they are to be believed, it happens also on the advice of child health workers including paediatricians. In older children - who are spoon fed - the most abusive tactic I have encountered is for the child's nose to be grasped firmly so as to provoke mouth breathing, for the food to be shovelled into the mouth that is then held shut, and for the throat to be stroked in a downward motion so as to 'encourage' swallowing. Not surprisingly, the child invariably finds the procedure intensely distressing and comes to regard feeds as occasions that are best avoided. A chain of association becomes built up in the child's mind between any hint that food is about to be presented, discomfort and fear. At first distress may be induced only as the food is brought near to the child's face, which is then averted, lips tightly shut. If the force feeding persists, as is often the case, this avoidance response is evoked by stimuli that are less and less directly related to the presentation of food. The child will struggle as he or she is placed in a highchair, requiring increasingly firm control. Occasionally the excessive force applied to strap the child in will fracture a clavicle, which is picked up by chance some time later on a chest radiograph that has been done for another purpose.

\section{Coaxing}

Coaxing a child to eat by persistently proffering food, even after repeated refusals, or allowing the child to determine the unique and bizarre circumstances in which he or she will deign to have a meal, is unwise. I have known of children who would only eat, for instance, sitting on top of a refrigerator in the kitchen so as to look out of the window and 'watch the birdies'. Playing elaborate distracting games, such as 'aeroplanes', seems to deplete appetite in the long term rather than increase it; they provide excellent examples of how even a very young child may condition the behaviour of a parent. We have ourselves observed mothers following their offspring around the room with food held out on a spoon, sedulously attempting to persuade the child to 'try a mouthful; just for mummy'.

Mealtimes are for eating and should be time limited; 20 minutes is a reasonable maximum for preschool children. To begin with, only the foods the child is known to accept should be presented. Small quantities of different foods, each presented separately, are better accepted than large quantities thrown together on one plate. Refusal should be ignored; as has already been emphasised, neither coaxing nor forcing will be effective in the long term. Social reinforcement (that is praise) is a crucial component of any treatment programme and works with the great majority of children. Undesired behaviours are not rewarded of course. If the child deliberately spits out food, or throws it on the floor, this is likely to be an attention seeking tactic. The central tenet of the recommended approach to managing feeding difficulties is to reward only feeding behaviour and, so far as possible, to ignore everything else, if necessary by looking in another direction so as to avoid eye contact with the miscreant. Children who are persistently poor feeders just do not seem to be as motivated by appetite as the rest of us. They will get just as much pleasure from successfully demanding social attention during mealtimes (directed at controlling their naughty behaviour) as they do from eating, and perhaps more. If success is encountered it may be advantageous to permit frequent closely spaced meals, both to increase the overall nutritional intake and to allow frequent exposure to positive reinforcement. Social reinforcement may be enhanced by the family at large, so the child should be encouraged to eat with other family members. Everybody joins in praising the child for eating a morsel! In many cases participant observation of normal peer feeding behaviour serves as a powerful motivator for change; seemingly intractable feeding problems often resolve when the self feeding child has an opportunity to eat at the nursery's communal lunch. ${ }^{17}$ Peer group modelling is not only effective in encouraging children to eat who are otherwise reluctant to accept anything, it is also a potent way of increasing the range of accepted foods. Most normal children like to copy the things they see their friends doing. Children who are eating well in such a communal situation are also receiving positive social reinforcement for their behaviour. They are learning gradually that eating can be fun, and that it is a social experience. Another technique to increase the range of accepted foods is to allow a child to take food from another's plate, be it parent, sibling, or friend. Children are especially attracted to foods they see others enjoying but the tactic will only work with relatively neutral foods for obvious reasons; if the child has a strong aversion to the foodstuff in question it will not be accepted.

\section{'One trial learning'}

Food refusal may develop on the basis of a learned aversion to certain foods, perhaps because they have in the past been associated with an unpleasant experience. If we eat something and vomit immediately afterwards we invariably assume, rightly or wrongly, that it was that particular food that was responsible. This is another example of what is teleologically an adaptive response, useful in the days when we were all hunter-gatherers. If $I$ ate a poisonous berry and it made me sick I wouldn't touch it again. What is so remarkable about this example of 'one trial' learning is the intensity of the avoidant response, which will be familiar to anyone who has experienced food poisoning. For young children with a medical condition, such as gastro-oesophageal reflux, which leads to vomiting after meals irrespective of what foods have been ingested, there is a serious danger that this potentially adaptive behaviour will be transmuted into a distinctly maladaptive response to any feeding occasion. For children receiving chemotherapy for cancer such one trial learning exacerbates the problem of a reduced appetite which is often already associated with the condition. 
Feeding disorders and failure to thrive

Children with minor organic disorders may fail to thrive, not because of any direct effect upon metabolism, but because the child is thereby very difficult to feed. For example, it has been shown that, within a group of otherwise healthy failing to thrive infants, significant degrees of oral motor dysfunction are common. ${ }^{18}$ Consequently, caretakers may be faced with a significant yet subtle and often unappreciated obstacle to ensuring their child ingests adequate nutrition for growth. Symptoms include hypotonic lips, incompetence removing food from a spoon, tongue thrust or persistent tongue protrusion, and a weak or unsustained suck. Unless the child is observed to eat by someone (for example, a speech therapist) who is trained to recognise such symptoms they will be missed.

Poor oral motor functioning may also be associated with impaired growth in infants and young children who suffer from conditions that are known as growth problems but in which the growth disorder is not thought to have been caused by a nutritional deficiency. For example, Mathisen et al, in a small series, found virtually all infants with Turner's syndrome had severe oral motor difficulties, including hypotonia of the orofacial musculature, some degree of tongue dysfunction (such as poor tongue tip differentiation and absent lateralisation), and incompetent chewing. ${ }^{19}$ Suck-swallow sequences were poorly coordinated, leading to gagging and choking. Failure to thrive was usual, and many had received non-oral feeds in order to treat that condition.

Failure to thrive is also commonly found in infants and young children with cerebral palsy. All too often the associated growth disorder has been ascribed to underlying neurological deficits rather than to chronic malnutrition. The reason why such children have difficulty achieving a nutritional intake sufficient to sustain a normal rate of growth includes a variety of characteristics that may well have relevance to the aetiology of some cases of failure to thrive in children with no overt organic disease or disorder. They include communication difficulties that inhibit or distort requests for food, impaired expression of hunger or food preferences, a lack of self feeding skills, an inability to forage, and severe degrees of oral motor dysfunction. Gastro-oesophageal reflux has been estimated to occur in up to $75 \%$ of subjects. ${ }^{20} \mathrm{In}$ a recent survey of domestic mealtime routines Reilly and Skuse found a sample of preschool children with cerebral palsy and known oral motor dysfunction had very severe feeding difficulties, which were for the most part unknown to their medical advisors. ${ }^{21}$ There was substantial evidence for gross malnourishment in most cases, associated with parental psychopathology that seemed to be contingent on the stress and strain of managing the children's feeding behaviour. Growth trajectories showed a dire picture with severe failure to thrive in the first few months of life. At that time, when demands for nutrition for physical growth were exceptionally high, ensuring an adequate intake was impossible for most caregivers.
During the first 12 months of life self regulation is an important determinant of mechanisms of appetite and satiety. ${ }^{22}$ As common experience shows, most infants are highly skilled letting their mothers know when they are hungry, usually by crying lustily, and also letting them know when they are satiated (by falling contentedly to sleep). Infant demand is a key determinant of lactation performance in mothers who are breast feeding. ${ }^{23}$ In other words, a mother's milk supply is directly related to the frequency and strength of her infant's suckling. It is important to be aware that infants who fail to thrive at the breast comprise two fairly distinct groups. Firstly, there are those who are restless, who cry excessively, who may not complete their feeds or who latch on inadequately to the nipple. ${ }^{24} \mathrm{~A}$ second, less well recognised group, include infants such as have been described by Evans and Davies. ${ }^{25}$ They appear to have little appetite, do not demand to be fed, and so may go excessively long periods between feeds. Typically parents regard these as very 'good babies' who sleep through the night from an early age and, even if they are woken for feeds, do not appear terribly interested in the idea.

Although 'feeding problems' are usually characterised as difficulties getting young children to take adequate nutrition, either through excessive faddiness or some other variety of food refusal, it is important to bear in mind that growth failure may occur as a consequence of a far more subtle interaction between infant characteristics and parental response. In a recent whole population survey of infants who were failing to thrive, within a disadvantaged inner city area, we found that about two out of three infants tended to 'sleep through feeds' (whether breast or bottle, before or after weaning) at some point in the first postnatal year. ${ }^{26}$ The great majority $(80 \%)$ of mothers in a well growing comparison group regularly woke their children on these occasions, but only $50 \%$ of mothers of failing to thrive babies said they did so.

In conclusion, feeding disorders in preschool children are relatively common both within and outside hospital practice. Management of such disorders is not usually based on an appreciation of the developmental course of normal feeding behaviour and appetite regulation. Consequently, many young children, whose problem is relatively minor to begin with, go on to develop intractable patterns of behaviour that not only cause anxiety and distress to their parents but may well have adverse consequences for their growth and health. Appropriately timed and organised primary and secondary prevention of feeding problems in children at risk could have major benefits for a wide variety of paediatric patients.

Minde K, Minde R. Infant psychiatry: an introductory text. London: Sage, 1986

2 Harris G, Booth IW. The nature and management of eating problems in preschool children. In: Cooper P, Stein A, ed. The nature and management of feeding problems and eating disorders in young people. Monographs in Clinical Paediatrics (Lanzkowsky P, ed.) New York: Harwood Academic, 1992: 61-84. 
3 Birch LL, Marlin DW. I don't like it: I never tried it: effects of exposure on two year old children's food preferences. Appetite 1982; 3: 353-60.

4 Harris G. Determinants of the introduction of solid food. Fournal of Reproductive and Infant Psychology 1988; 6: 241-9.

5 Jones VM. Current infant weaning practices within the Bangladeshi community in the London borough of Tower Hamlets. Human Nutrition Applied Nutrition 1987; 41a: 349-52.

6 Illingworth RS, Lister J. The critical or sensitive period, with special reference to certain feeding problems in infants and children. F Pediatr 1964; 65: 839-49.

7 Morris SE. Development of oral-motor skills in the neurologically impaired child receiving non-oral feedings. Dysphagia 1989; 3: 135-54

8 Birch LL. The control of food intake by young children: the role of learning. In: Capaldi ED, Powley TL, eds. Taste, experience and feeding. Washington DC: APA, 1990: 116-38.

9 Davis CM. Self-selection of diet by newly weaned infants: an experimental study. $\mathrm{Am} \mathcal{F}$ Dis Child 1928; 36: 651-79.

10 Davis CM. The self-selection of diet experiment: its significance for feeding in the house. Ohio State Medical fournal cance for feeding

11 Story M, Brown JE. Do young children instinctively know what to eat? N Engl f Med 1987; 316: 103-7.

12 Cowart BJ. Development of taste perception in humans: sensitivity and preference throughout the life span. Psychol Bull 1981; 90: 43-73.

13 Birch LL. Development of food acceptance patterns. Developmental Psychology 1990; 26: 515-9.

14 Beauchamp GK, Moran M. Acceptance of sweet and salty tastes in 2 year old children. Appetite 1984; 5: 291-305.
15 Birch LL, Birch D, Marlin D, Kramer L. Effects of instrumental eating on children's food preferences. Appetite 1982; 3: 125-34

16 Birch LL, Marlin DW, Rotter J. Eating as the 'means' activity in a contingency: effects of young children's food preference. Child Dev 1984; 55: 431-9.

17 Birch LL. Effects of peer models' food choices and eating behaviors on preschoolers' food preferences. Child Dev behaviors on presch

18 Mathisen B, Skuse D, Wolke D, Reilly S. Oral-motor dysfunction and failure to thrive amongst inner-city children.

19 Mathisen B, Reilly S, Skuse D. Oral-motor dysfunction and feeding disorders in infants with the Turner syndrome. Dev Med Child Neurol 1992; 34: 141-9.

20 Rempel GR, Colwell SO, Nelson RP. Growth in children with cerebral palsy fed via gastrostomy. Pediatrics 1988 82: 857-63.

21 Reilly S, Skuse D. Characteristics and management of feeding problems in young children with cerebral palsy. Dev Med Child Neurol 1992; 34: 379-88.

22 Pridham KF. Session III: infant feeding behavior and parenting. Feeding behavior of 6 to 12 months old infants: assessment and sources of parental information. Pediatrics 1990; 117: 174-80.

23 Dewey KG, Lonnerdal B. Infant self-regulation of breast milk intake. Acta Paediatr Scand 1986; 75: 893-8.

24 Hyett FE, Yorston JC, Thompson AM. Difficulties associated with breast feeding: a study of 106 primiparas. $B M \mathcal{F}$ 1958; i: 310-5.

25 Evans TJ, Davies DP. Failure to thrive at the breast: an old problem revisited. Arch Dis Child 1977; 52: 974.

26 Skuse D, Reilly S, Wolke D. Psychosocial adversity and growth during infancy. Eur $\mathcal{f}$ Clin Nutr 1993 (in press). 